\title{
CONF- $9410165-11$
}

Version: September 16, 1994

Recent Neutron Scattering Results on

High-Temperature Superconductors

H. A. Mook, ${ }^{1}$ P. Dai, ${ }^{1}$ G. Aeppli, ${ }^{2}$ T. E.

Mason, ${ }^{3}$ N. E. Hecker, ${ }^{4}$ J. A. Harvey, ${ }^{4}$

T. Armstrong, ${ }^{5}$ K. Salama, ${ }^{6}$ D. Lee $^{6}$

1. Oak Ridge National Laboratory, P. O. Box 2008, Oak Ridge TN 378316393, USA

2. AT\&T Bell Laboratories, Murray Hill, NJ 07974, USA

3. University of Toronto, $60 \mathrm{St}$. George St., Toronto, Ontario, Canada

4. Harvard University, Cambridge, MA 02138, USA

5. Battelle Northwest Laboratories, Richland, WA, USA

6. Texas Center for Superconductivity, University of Houston, Houston, TX 77204

\section{Abstract}

Triple-axis spectrometry has been used to determine the magnetic excitations in $\mathrm{YBa}_{2} \mathrm{Cu}_{3} \mathrm{O}_{7}$. Polarized measurements at $100 \mathrm{~K}$ show the scattering consists of a peak near $40 \mathrm{meV}$ superposed on a small relatively flat background. The $40-\mathrm{meV}$ peak is found to be sharp in energy and anisotropic in momentum occupying a square portion of the $\mathrm{Cu}-\mathrm{O}$ reciprocal lattice plane. The temperature dependence of the scattering is identical at the square side and corner. Polarized measurements show that the small background scattering observed at $100 \mathrm{~K}$ disappears below the superconducing transition. Resonant absorption measurements made to examine the $\mathrm{Cu}$ phonons show that no changes in the phonons occur at the superconducting transition. 
Keywords: High- $T_{\mathcal{C}}$ Temperature Superconductivity, Magnetic Excitations, Phonons

\author{
H. A. Mook
}

Solid State Division

Oak Ridge National Laboratory

P. O. Box 2008

Oak Ridge, TN 37831-6393, USA

FAX: (615)574-6268; email: HAM@ornl.gov

\title{
DISCLAIMER
}

This report was prepared as an account of work sponsored by an agency of the United States Government. Neither the United States Government nor any agency thereof, nor any of their employees, makes any warranty, express or implied, or assumes any legal liability or responsibility for the accuracy, completeness, or usefulness of any information, apparatus, product, or process disclosed, or represents that its use would not infringe privately owned rights. Reference herein to any specific commercial product, process, or service by trade name, trademark, manufacturer, or otherwise does not necessarily constitute or imply its endorsement, recommendation, or favoring by the United States Government or any agency thereof. The views and opinions of authors expressed herein do not necessarily state or reflect those of the United States Government or any agency thereof. 


\section{DISCLAIMER}

Portions of this document may be illegible in electronic image products. Images are produced from the best available original document. 
There has been a large number of neutron studies on high-temperature superconductors that have yielded very important results concerning several aspects of the high- $T_{\mathcal{c}}$ problem. These would be difficult to cover even in a lengthly manuscript, so we will limit ourselves to recent neutron scattering measurements on $\mathrm{YBa}_{2} \mathrm{Cu}_{3} \mathrm{O}_{7}$ (Y123). Most of the measurements concern the magnetic fluctuations for the material; however, some measurements on the lattice dynamics will be mentioned at the end of the article.

The measurements on the magnetic excitations have been performed on large crystals of Y123 that range from 8-15 grams in weight and have mosaic spreads on the order of $1.5^{\circ}$. Most of the measurements were made on two crystals mounted together giving a combined weight of about 20 grams. The crystals contain a considerable amount (15\%) of $\mathrm{Y}_{2} \mathrm{BaCuO}_{5}$ as an impurity. This material is found in large pores of the crystals and is oriented randomly with respect to the crystal lattice. We have studied the temperature dependence of the scattering of a large powder sample of $\mathrm{Y}_{2} \mathrm{BaCuO}_{5}$ to make sure that none of the effects which we ascribe to $Y 123$ actually occur in the impurity phase. The crystals contain no other phases of significance. It is 
difficult to ascertain the exact oxygen concentration of such large crystals, but bulk measurements on similar smaller crystals result in oxygen concentrations very near $\mathrm{O}_{7}$. Lattice constant measurements for the crystals confirm that the entire sample has an oxygen concentration consistent with the $\mathrm{O}_{7}$ value. The crystals show superconducting transitions at $92.4 \mathrm{~K}$ that are $1-\mathrm{K}$ wide. Measurements of the vortex lattice have been made with the crystals used in the experiment, and the intensity of the scattering from the vortex lattice[1] shows that the entire crystal exhibits bulk superconductivity.

Figure 1 shows the reciprocal lattice of the Y123 for the $a^{*}, b^{*}$ directions shown in square lattice notation. Our crystals are all highly twinned so that we cannot distinguish $a^{*}$ from $b^{*}$ in our measurements. We find the magnetic response to center on $\Pi, \Pi$, and we have performed numerous scans in the direction $(0,0$ to $2 \Pi, 2 \Pi)$ which we will denote as $(\Pi, \Pi)$ scans and along the direction $(0, \Pi$ to $2 \Pi, \Pi)$ which we will denote as $(0, \Pi)$ scans.

We have found the phonon scattering in the sample to be much larger than the magnetic scattering in some cases. This means that when a scattering signal is found, polarization analysis 
must be used to confirm that the scattering is magnetic in nature. If the unpolarized scan results in no signal being observed, it can be relied on to prove that no excitation of any type exists in the region being probed or that the scattering is small and featureless and thus difficult to distinguish from the background. For polarized scans it is often sufficient to measure the spin-flip scattering (SF) and the non-spin flip scattering (NSF). The SF will give the background plus magnetic scattering, while the NSF will give the background plus nonmagnetic scattering. Generally the background can be established to be quite flat so that a peak in the SF scattering, corrected for any imperfect polarization effects, can be assumed to be magnetic. In questionable cases or where the scattering is flat, the SF scattering can be measured with the neutron polarization first parallel to the neutron momentum transfer (HF) and then perpendicular (VF). The difference HF-VF will then give one half the magnetic intensity completely free of background effects.[2] This type of measurement is very reliable since only the field changes direction, and all background effects cancel. Polarized measurements result in a loss in intensity of a factor between five and ten, and the HF-VF case is much more difficult yet as two 
measurements are needed which result in one half the intensity.

We find the dominant feature in the magnetic scattering to be a peak near $40 \mathrm{meV}$ as observed in earlier measurements.[3] Figure 2 shows a $(\Pi, \Pi)$ polarized, HF-VF, scan taken at $100 \mathrm{~K}$. All our scans shown were taken with a $c$-axis component on the peak of the bilayer structure factor, [4] so that the scattering can be thought of as stemming from acoustic magnetic excitations. Since we have done a HF-VF measurement, the only scattering observed is magnetic, and little scattering is found except for the 40-meV peak. We will show later that there is a small featureless magnetic intensity in addition to the $40-\mathrm{meV}$ peak. We find the peak is narrow in energy (equal to our resolution) even at $100 \mathrm{~K}$. Earlier measurements have shown that the peak narrows in momentum $q$ as the temperature is lowered and the peak height grows correspondingly.

Previous polarized measurements[3] have shown that the phonons do not change much between $100 \mathrm{~K}$ and $10 \mathrm{~K}$ so that we can examine the $40-\mathrm{meV}$ scattering with unpolarized neutrons if we employ temperature differences. Figure 3a shows a $(0, \Pi)$ result through the peak obtained by subtracting $100 \mathrm{~K}$ from $10-\mathrm{K}$ data. We see the peak 
is about 0.24 momentum units wide along the scan direction, while Fig. $3 b$ shows the result for a $(\Pi, \Pi)$ scan in which the peak is essentially the same width in units of the scan, or $\sqrt{2}$ wider in momentum units. The actual peak width must be obtained from a deconvolution of the data with the spectrometer resolution. The scan directions and the size and position of the resolution ellipses are shown in Fig 1 . The resolution corrected widths are $0.35 \AA^{-1}$ for the $(0, \Pi)$ scan and $0.49 \AA^{-1}$ for the $(\Pi, \Pi)$ scan. The $40-\mathrm{meV}$ feature is thus not circular around $(\Pi, \Pi)$ as would be expected for a conventional spin-wave excitation with a dispersion governed by a simple exchange interaction. The feature instead appears to have the square symmetry of the reciprocal lattice and thus be associated with the Fermi surface. The size of the area covered by the $40-\mathrm{meV}$ feature relative to the square zone is shown as the smaller square in Fig 1.

Since the scattering appears to result from a Fermi surface effect, it is interesting to examine the temperature dependence of the $40-\mathrm{meV}$ feature and to see if there is any difference in its behavior at the half width position at the square corner in the $(\Pi, \Pi)$ direction from the half width position at the square edge in the $(0, \Pi)$ direction, as might 
occur from anisotropic superconducting pairing. Figure $4 \mathrm{a}$ and $\mathrm{b}$ show the result for the two directions. The data shown has the background scattering subtracted from it as determined by a measurement at the zone boundary. The temperature dependence of the feature measured at $(\Pi, \Pi)$ given by the open circles must be the same in both measurements and agree with the earlier measurement.[4] This is in fact the case. The points denoted by the squares are taken near the half widths of the scattering distributions at $10 \mathrm{~K}$ for the two directions. The absolute $q$ value is $\sqrt{2}$ larger for $(\Pi, \Pi)$ scan confirming the square symmetry of the distribution. We note that the scattering intensity at the position of the half width at $10 \mathrm{~K}$ is independent of temperature. The scattering distribution thus narrows upon cooling and increases in intensity in such a way that the intensity at the $10 \mathrm{~K}$ half-width value is nearly unchanged. This occurs in the same way for both scan directions showing that the temperature dependence $41-\mathrm{meV}$ feature is insensitive to anisotropic pairing if it occurs.

Although Fig. 2 shows that the main contribution to the scattering occurs at $40 \mathrm{meV}$, closer inspection reveals that a small additional magnetic intensity is observed that is 
$q$ independent. No sharp features have been found in the scattering other than the one at $40 \mathrm{meV}$. Figure 5 shows scans made at $11 \mathrm{meV}$ in order to study the lower energy scattering. Scans at this energy are found to be relativity free of phonon scattering; however, polarized measurements are a necessity to determine the magnetic scattering. Figure 5a shows a $(\Pi, \Pi)$ polarized (HF) scan at $11 \mathrm{meV}$ showing that the scattering is flat at $100 \mathrm{~K}$, while Fig. $5 \mathrm{~b}$ shows a similar result at $35 \mathrm{~K}$. We note the intensity is slightly smaller at $35 \mathrm{~K}$, but detailed measurements using the HF-VF polarized technique are necessary to isolate this effect from temperature dependent background effects. Figure $5 \mathrm{c}$ shows a nonpolarized $(0, \Pi)$ scan at $11 \mathrm{meV}$. If any structure existed similar to that observed for $\mathrm{La}_{1-\delta} \mathrm{Sr}_{\delta} \mathrm{CuO}_{4},[5]$ it would be apparent in this scan. However, we find the scan again to be flat. Since this scan passes through the point $(\Pi, \Pi)$ as do the scans shown in Fig. $4 a$ and $b$, the intensity must be identical in these directions at least at $100 \mathrm{~K}$, as all scans show no structure.

In order to determine the magnetic contribution to the scattering HF-VF, polarized measurements have been made at $(\Pi, \Pi)$ at $11 \mathrm{meV}$. Figure 6 shows the temperature 
dependence of the magnetic scattering. A small but observable contribution is found at $100 \mathrm{~K}$ so that a uniform magnetic background is found at this temperature. As the temperature is lowered, this scattering is found to disappear. No scattering is observed at the lowest temperatures within the experimental error. Polarized measurements are absolutely essential for this measurement, as even though the nonmagnetic scattering is relatively small, it is about 250 counts at $11 \mathrm{meV}$ on the scale of the figure. The beam polarization was monitored through the superconducting transition and found not to be affected adversely. The temperature dependence of the susceptibility is similar to that observed in NMR spin-lattice relaxation time measurements for the planer $\mathrm{Cu}$ sites.[6] However, the large enhancement found for the $\mathrm{Cu}$ sites relative to the O sites may be more difficult to explain given the flat nature of the scattering. Of course much of reciprocal space remains unexplored, and sharp structure may occur that has not yet been found.

We conclude our discussion of neutron results for Y123 with a brief mention of recent measurements of the lattice dynamics. Earlier measurements using neutron resonance absorption spectroscopy (NRAS) had shown that 
the $\mathrm{Cu} a-b$ plane modes displayed an anomalous softening at a temperature slightly higher than the superconducting transition for $\mathrm{Bi}_{2} \mathrm{Sr}_{2} \mathrm{CaCu}_{2} \mathrm{O}_{8}$.[7] The NRAS measurements measure the kinetic energy (KE) of the $\mathrm{Cu}$ phonons. The $\mathrm{KE}$ is given by the integral of the $\mathrm{Cu}$ phonon density of states weighted by the phonon energy. The measurement examines the $\mathrm{Cu}$ phonons in all directions in the $a, b$ plane and is more sensitive to the higher energy phonons. The $\mathrm{Bi}_{2} \mathrm{Sr}_{2} \mathrm{CaCu}_{2} \mathrm{O}_{8}$ measurement and the presumed observation of large changes in the ion channeling for $\mathrm{ErBa}_{2} \mathrm{Cu}_{3} \mathrm{O}_{7}[8]$ near $T_{c}$ suggested that phonons might play an important role in the superconductivity. We have now made NRAS measurements for $\mathrm{Y} 123$ for both $\mathrm{O}_{7}$ and $\mathrm{O}_{6}$ compositions. The new measurements show no change in the $\mathrm{Cu} K E$ at $T_{c}$ and no substantial softening of the phonons above $T_{\mathcal{c}}$, as shown in Fig. 7. The phonon behavior found for $\mathrm{Bi}_{2} \mathrm{Sr}_{2} \mathrm{CaCu}_{2} \mathrm{O}_{8}$ is thus not essential for superconductivity. One interesting feature found in the NRAS measurements for both Y123 and $\mathrm{Bi}_{2} \mathrm{Sr}_{2} \mathrm{CaCu}_{2} \mathrm{O}_{8}$ is the lack of any drop off in the $\mathrm{Cu} \mathrm{KE}$ below $T_{c}$. This shows that the $\mathrm{Cu}$ phonons must harden below $T_{\mathcal{C}}$, which is opposite to the behavior expected for a conventional 
explanation of the superconductivity in terms of phonons. This of course provides no proof that phonons are not of primary importance for high- $T_{c}$ superconductivity but does diminish direct evidence that this is the case.

\section{ACKNOWLEDGMENT}

Research supported in part by the Division of Materials Sciences, U.S. Department of Energy, under Contract No. DE-AC05-84OR21400 with Martin Marietta Energy Systems, Inc.

References:

[1] M. Yethiraj, H. A. Mook, G. D. Wignall, R. Cubitt, E. M. Forgan, D. M. Paul, and T. Armstrong, Phys. Rev. Lett. 70 (1993) 857.

[2] R. M. Moon, T. Riste, and W. C. Koehler, Phys. Rev. 181 (1969) 920.

[3] H. A. Mook, M. Yethiraj, G. Aeppli, T. E. Mason, and T. Armstrong, Phys. Rev. Lett. 70 (1993) 3490.

[4] J. M. Tranquada, P. M. Gehring, G. Shirane, S. Shamoto, and M. Sato, Phys. Rev. B 46 (1992) 5561.

[5] S-W. Cheong, G. Aeppli, T. E. Mason, H. Mook, S. M. Hayden, P. C. Canfield, Z. Fisk, K. N. Clausen, and J. L. Martinez, Phys. Rev. Lett. 67 (1991) 1791.

[6] R. E. Walstedt and W. W. Warren, Jr., Science 248 (1990) 1082. 
[7] H. A. Mook, M. Mostoller, J. A. Harvey, N. W. Hill, B. Chakoumakos, and B. C. Sales, Phys. Rev. Lett. 69 (1992) 2272.

[8] R. P. Sharma, L. E. Rehn, P. M. Baldo. and J. Z. Liu, Phys. Rev. Lett. 69 (1992) 2275. 
Figure Captions:

1. Diagram of the 2-D Magnetic Brillouin Zone in square lattice notation. The smaller square gives the half width of the scattering found at about $40 \mathrm{meV}$, and the ellipses show the resolution used in the measurement.

2. Polarized beam determination of the energy dependence of the magnetic scattering at $(\Pi, \Pi)$. The measurements were made by the HF-VF technique which isolates only the magnetic scattering. There is a region centered around $25 \mathrm{meV}$ where large parasitic scattering from the $(0,0,6)$ reflection makes measurements impossible with the crystal orientation used.

3. $(0, \Pi)$ scans and $(\Pi, \Pi)$ scans through the feature found near $40 \mathrm{meV}$. The data are the diffrence from a measurement taken at $10 \mathrm{~K}$ and one taken at $100 \mathrm{~K}$.

4. Temperature dependence of the scattering from the $40-\mathrm{meV}$ feature. Mesurements were taken in crystal orientations to sample the scattering at the reciprocal lattice square corner and edge. The circles give the scattering at $(\Pi, \Pi)$ for the two orientations while the squares give the intensity near the half width of the $10-\mathrm{K}$ distributions. Background scattering found at the zone boundry has been subtracted from the data.

5. Measurements made at $11 \mathrm{meV}$ to sample the small magnetic scattering found at all energies in addition to the $40-\mathrm{meV}$ peak. The measurements are for scans through $(\Pi, \Pi)$ along the zone diagonal and parallel to the zone edge. 
6. Temperature dependence of the scattering at $(\Pi, \Pi)$ for an energy transfer of $11 \mathrm{meV}$. The measurements are HF-VF polarized results which isolate only the magnetic scattering. Several measurements were averaged together resulting in the error bars shown.

7. Kinetic energy of the $\mathrm{Cu}$ phonons as a function of temperature. The square points are for the $\mathrm{O}_{7}$ composition and the diamond points for $\mathrm{O}_{6.2}$. The round points are for the sample reoxygenated to the $\mathrm{O}_{7}$ composition. 


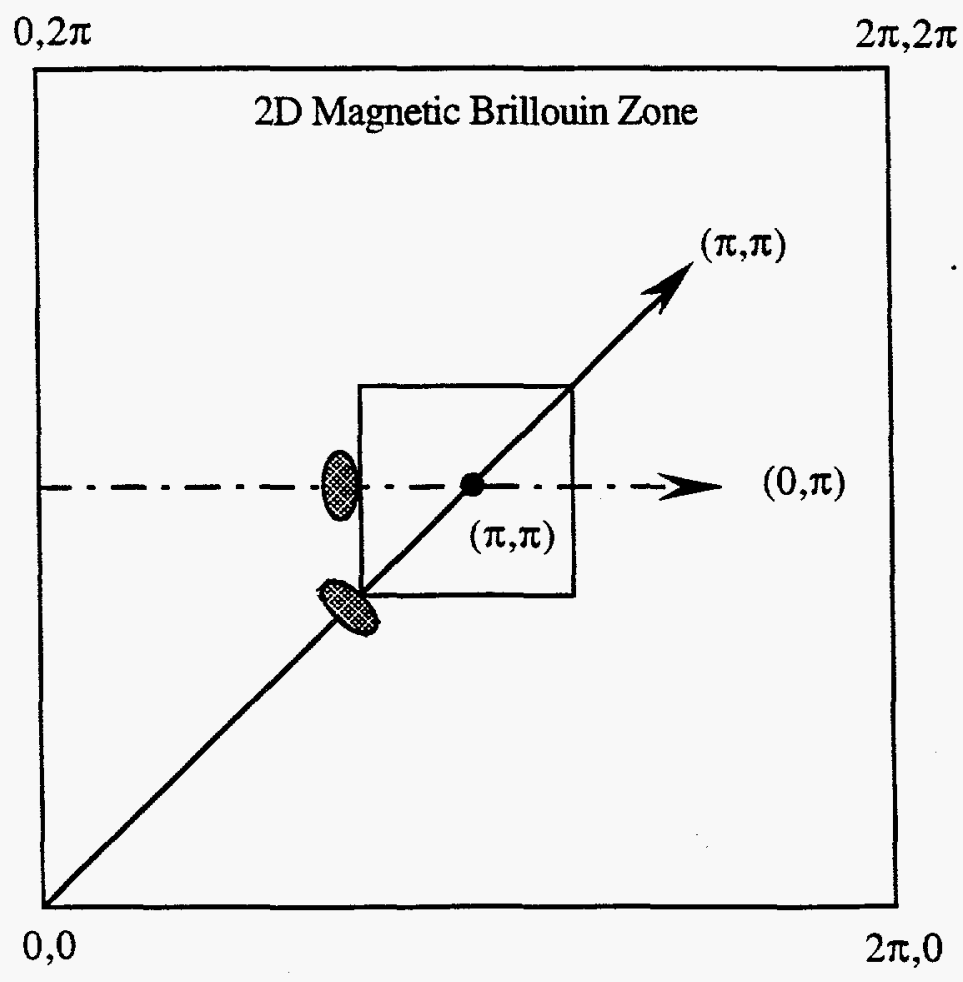


Recent Neutron Scattering Results on High-Temperature Superconductors

H. A. Kook, P. Dat, G. Aeppli, T. E. Mason, N. E. Hecker, J. A. Harvey, and T. Armstrong

Figure 1 


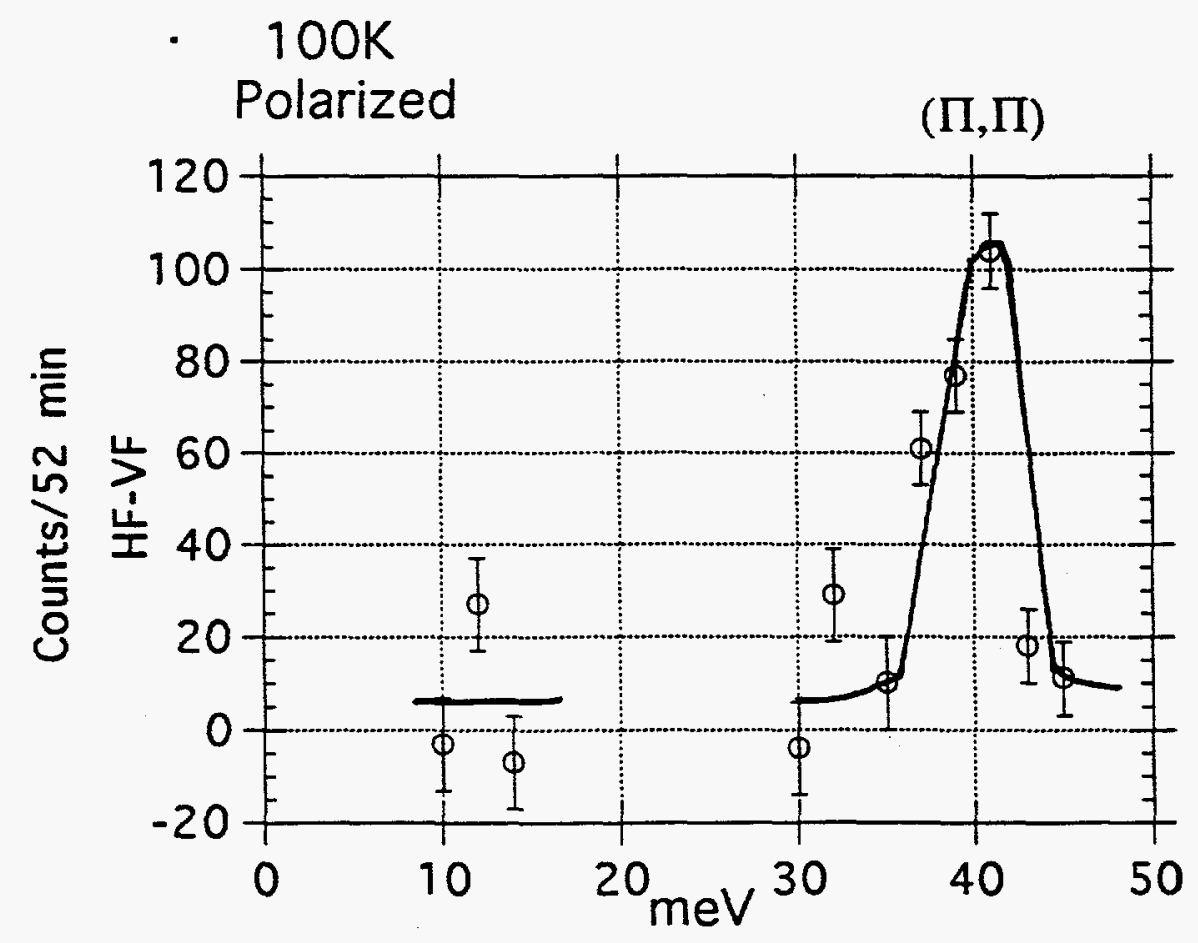


Recent Neutron Scattering Results on High-Temperature Superconductors

H. A. Mook, P. Dai, G. Aeppli, T. E. Mason, N. E. Hecker,

Figure 2 

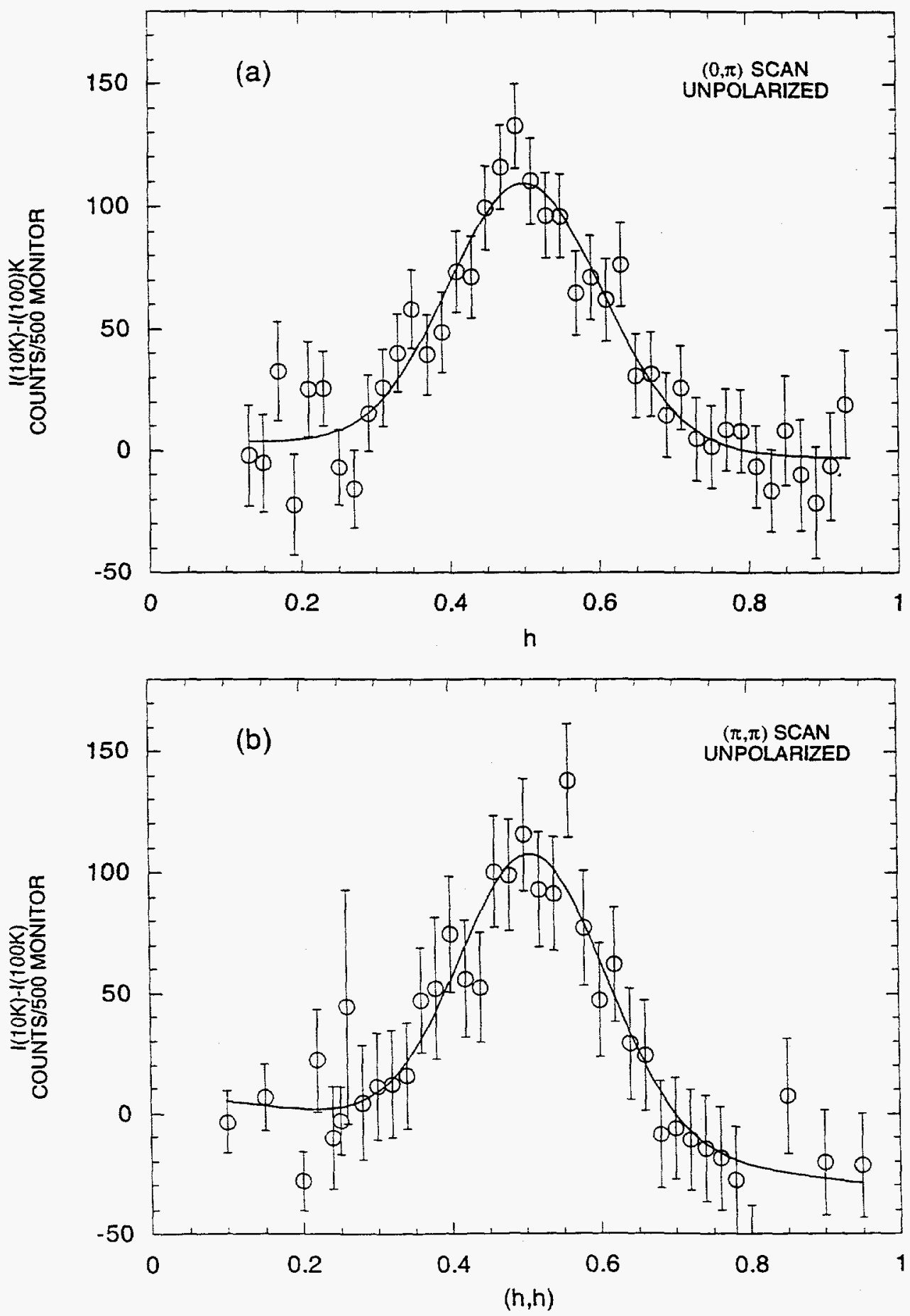
Recent Neutron Scattering Results on High-Temperature Superconductors

H. A. Mook, P. Dai, G. Aeppli, T. E. Mason, N. E. Hecker, J. A. Harvey, and T. Armstrong

Figure 3 

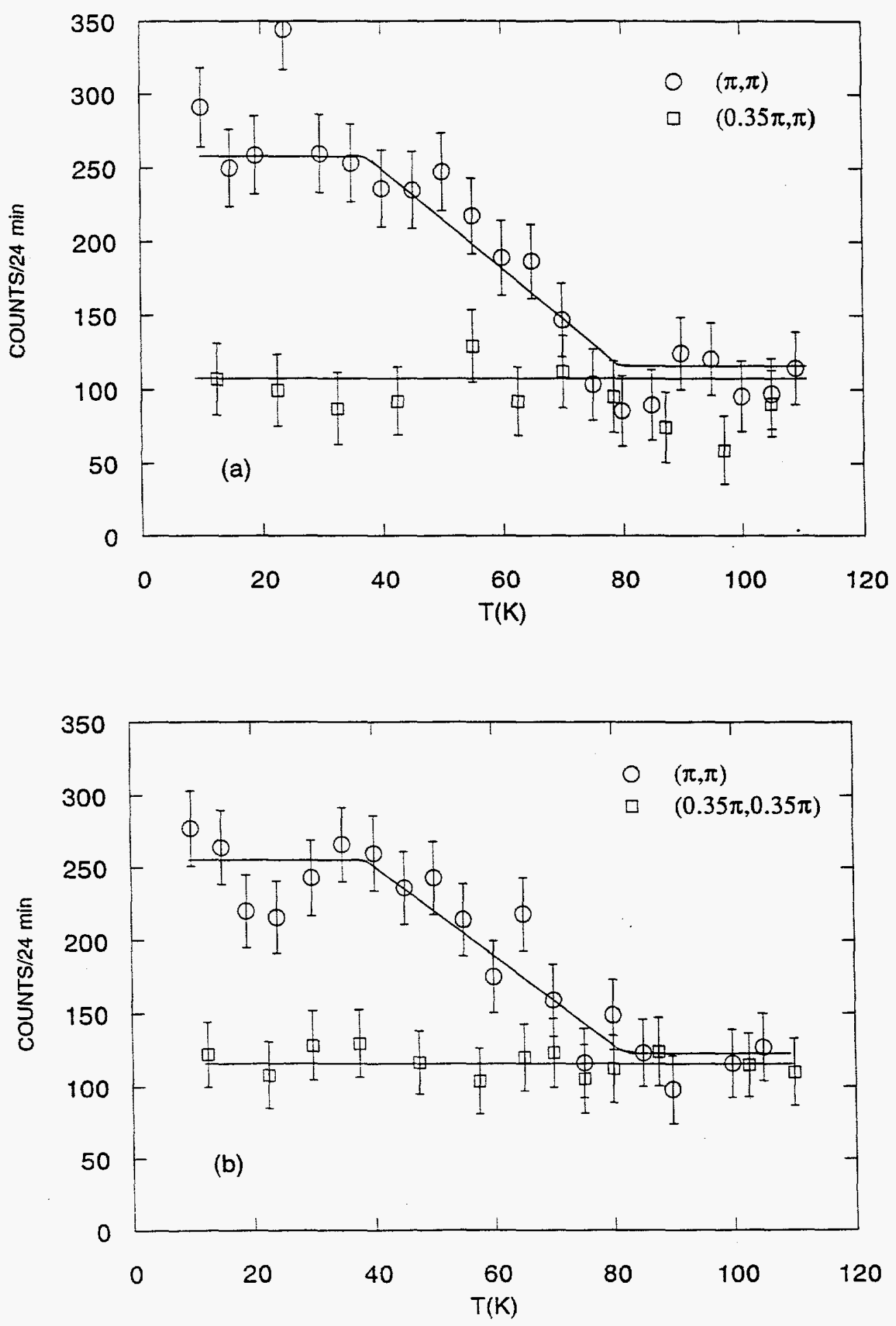
Recent Neutron Scattering Results on High-Temperature Superconductors

H. A. Mook, P. Dai, G. Aeppli, T. E. Mason, N. E. Hecker,

Figure 4 

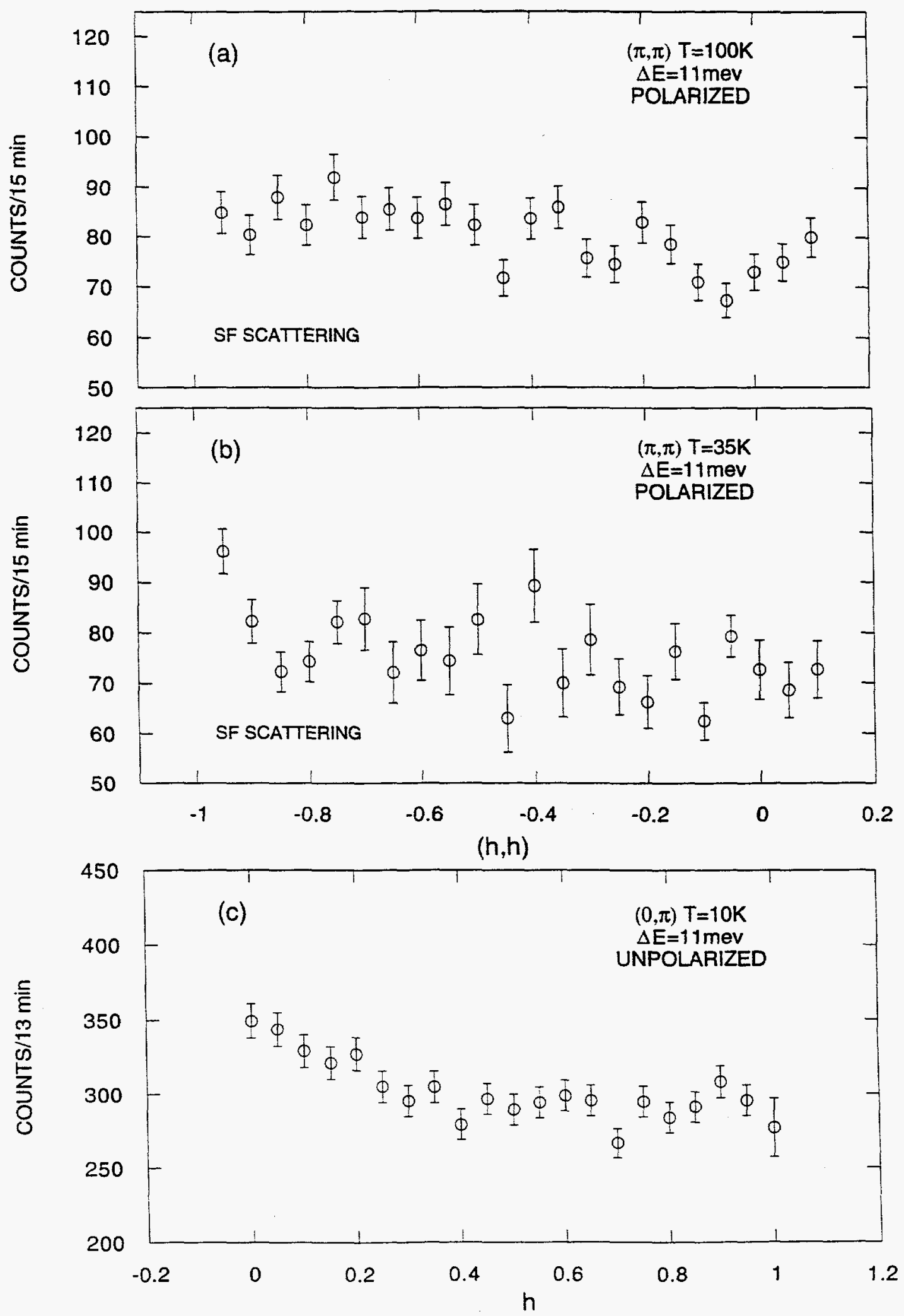
Recent Neutron Scattering Results on High-Temperature Superconductors

H. A. Mook, P. Dai, G. Aeppli, T. E. Mason, N. E. Hecker, J. A. Harvey, and T. Armstrong

Figure 5 


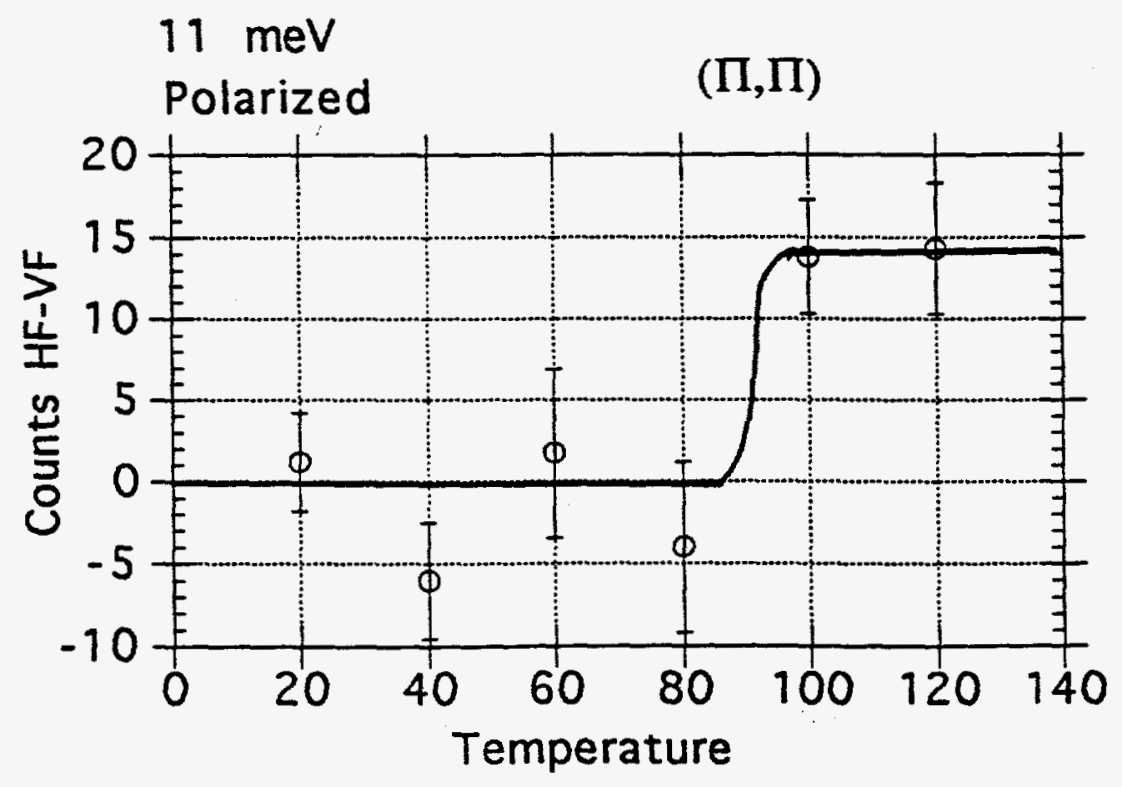


Recent Neutron Scattering Results on High-Temperature Superconductors

H. A. Mook, P. Dai, G. Aeppli, T. E. Mason, N. E. Hecker,

Figure 6 


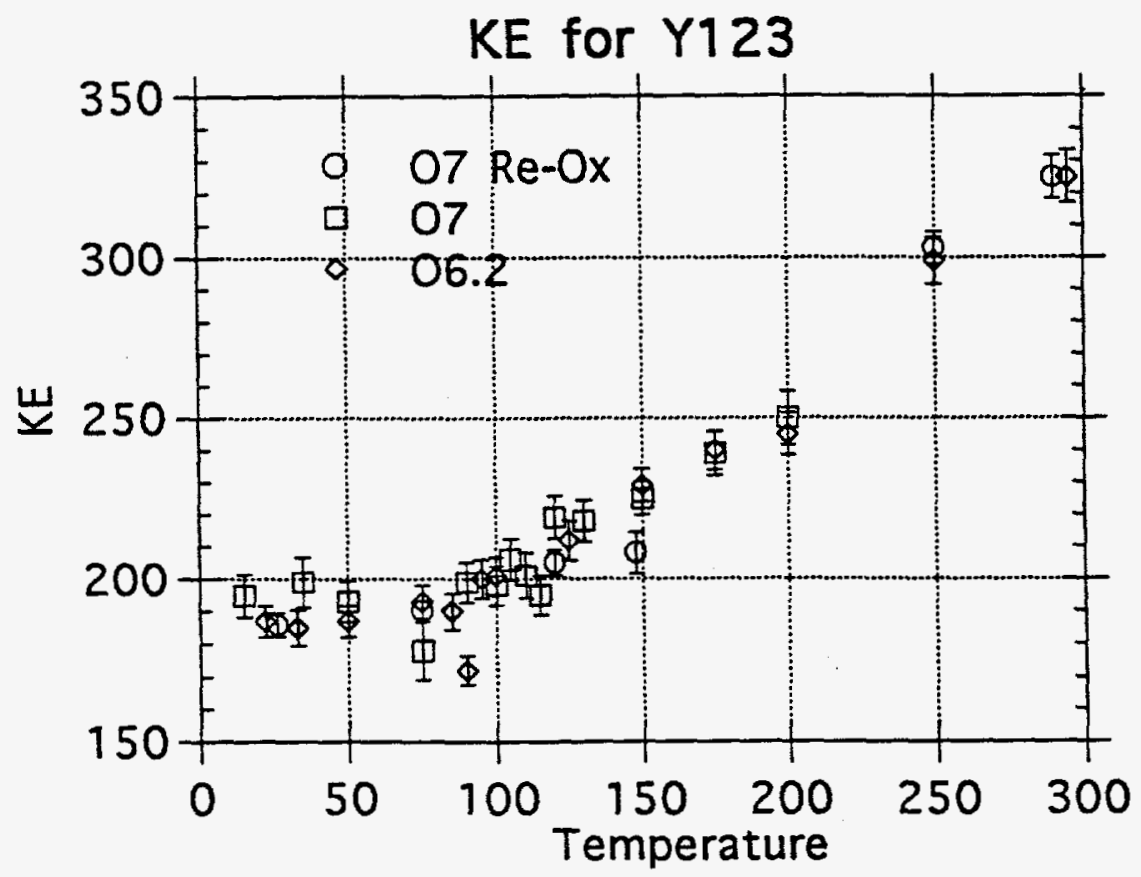


Recent Neutron Scattering Results on High-Temperature Superconductors

H. A. Mook, P. Dai, G. Aeppli, T. E. Mason, N. E. Hecker,

J. A. Harvey, and T. Armstrong

Figure 7 